\title{
BOWEN RESONANCE FLUORESCENCE LINES OF OIII IN
}

\author{
PLANETARY NEBULAE \\ C. R. O'DELL and C. O. MILLER \\ RICE UNIVERSITY \\ P. O. Box 1892 \\ Houston, Texas, USA
}

\begin{abstract}
New and published line intensities are used to test the role of the pumping of the Bowen Lines of $\mathrm{O}$ III by absorption of the O1 and $\mathrm{O} 3$ lines and population by Charge Exchange. Although general agreement is obtained, noticeable deviations from theory exist.
\end{abstract}

We have obtained uniquely complete observations down to the ozone cutoff of the atmospheric window of the Bowen Resonance Fluorescence Lines of O III in the moderate ionization planetary nebula NGC 6210 and the high-ionization objects NGC 7027 and 7662 (O'Dell \& Miller 1992). These data are added to an additional eight well measured high-ionization nebulae (Likkel \& Aller 1986). Comparison of the observed lines with those predicted theoretically for the dominant O1 excitation shows general agreement, although some disagreements are present. We also measured lines arising from pumping by the secondary O3 line, which occurs farther away from the center of the He II Ly $\alpha$ line, and find good agreement with 20 year old theoretical predictions (Harrington 1972) for NGC 7027 and 7662 but unexpectedly high pumping rates in NGC 6210 . The one unblended line resulting only from $\mathrm{O} 3$ pumping may, however, be underestimated due to atmospheric ozone absorption. Examination of the role of Charge Exchange in explaining the excess of radiation from certain levels indicates that the process is important but does not quantitatively agree with theoretically predicted rates (Sternberg et al. 1988, Gargaud et al. 1989) for transitions out of the ${ }^{3} \mathrm{~S}_{1}$ state. If the O3 pumping has been underestimated, the ${ }^{3} \mathrm{~S}_{1}$ discrepancy disappears. The high-ionization nebulae are all very similar and stand in contrast with NGC 6210 , which shows unexpectedly high rates of pumping by $\mathrm{O} 3$ and population by Charge Exchange. These differences are probably due to different distribution within the nebula of the pumping line (He II $\mathrm{Ly} \alpha)$, O III, and the key ingredient in Charge Exchange $\left(\mathrm{H}^{0}\right)$.

\section{References}

Gargaud, I., McCarroll, R., \& Opradolce, L.: 1989, $A \& A$ 208, 251

Harrington, J. P.: 1972, ApJ 176, 127

Likkel, L. \& Aller, L. H.: 1986, ApJ 301, 825

O'Dell, C. R. \& Miller, C. O.: 1992, ApJ 390, 219

Stemberg, A., Dalgamo, A., \& Roueff, E.: 1988, Comm. Ap. 13, 29 